\title{
Erratum to: Silymarin attenuated mast cell recruitment thereby decreased the expressions of matrix metalloproteinases- 2 and 9 in rat liver carcinogenesis
}

Gopalakrishnan Ramakrishnan • Sundaram Jagan • Sattu Kamaraj • Pandi Anandakumar •

Thiruvengadam Devaki

Published online: 31 October 2012

(C) Springer Science+Business Media New York 2012

Erratum to: Invest New Drugs 2009; 27(3):233-240

DOI 10.1007/s10637-008-9163-y

In the original version of the article Figures 3 and 4 represents the immunohistochemical staining of MMP-2 \& MMP-9 respectively. But inadvertently both the images are same. We noticed the error and found it is the immunohistochemical staining of MMP-9 (Fig. 4) which is repeated in the Fig. 3. The correct Figure 3 is provided below.

The online version of the original article can be found at doi: http:// dx.doi.org/10.1007/s10637-008-9163-y.

G. Ramakrishnan · S. Jagan · S. Kamaraj • P. Anandakumar •

T. Devaki $(\bowtie)$

Department of Biochemisty, University of Madras,

Guindy Campus,

Chennai 600025 Tamilnadu, India

e-mail: biorkn@gmail.com 
Fig. 3 Representative

immunohistochemical staining of MMP-2 in the liver of control and experimental animals at 40x of original magnification. Plate $1-5$ represent the liver sections of groups $1-5$ of experimenal animals respectively. $\rightarrow$ shows immunostained areas
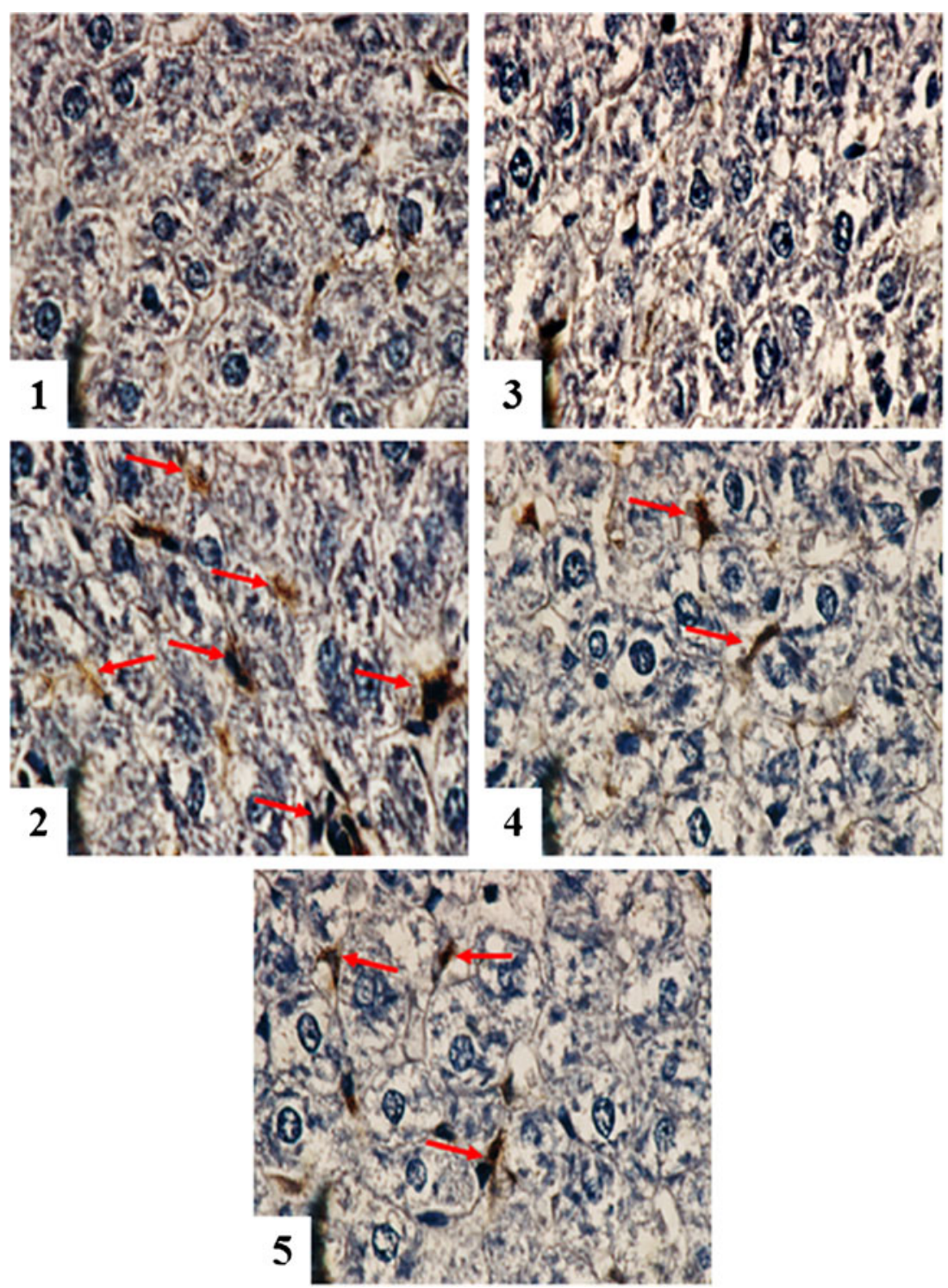